\title{
Plan de vaccination suisse 2012
}

\author{
Le plan de vaccination suisse 2012 (Directives et recommandations) vient d'être \\ publié [1]. Les nouveautés ou adaptations principales sont décrites ci-dessous.
}

Office fédéral de la santé publique, Commission fédérale pour les vaccinations

\section{Nouveautés et adaptations 2012}

Diphtérie et tétanos [2]

Vaccination recommandée de base (adultes) Dans le but de limiter les doses recommandées au minimum nécessaire, la Commission fédérale pour les vaccinations (CFV) et l'Office fédéral de la santé publique (OFSP) recommandent, pour les adultes de 25 à 64 ans d'allonger l'intervalle entre les rappels de vaccination diphtérie $(\mathrm{d})$ - tétanos $(\mathrm{T})$ de 10 à 20 ans. Les rappels dT doivent donc être faits à 25,45 et 65 ans puis, comme par le passé, tous les dix ans.

Vaccinations recommandées pour des groupes/ situations à risque

En cas de prophylaxie antitétanique lors de plaie l'intervalle des rappels dT pour les personnes âgées de 25 à 64 ans est allongé de 10 à 20 ans respectivement de 5 à 10 ans selon la gravité et le type de blessure. Les intervalles de rappel antitétaniques lors de plaie pour les adultes âgés de moins de 25 ans, de 65 ans et plus, et pour les personnes immunodéficientes restent inchangés (respectivement 5 et 10 ans selon le type de plaie/risque d'infection). Des intervalles plus courts que 20 ans (ou 10 ans à partir de 65 ans) peuvent être indiqués pour les voyageurs selon les circonstances à évaluer au cas par cas (par exemple région de haute endémie de diphtérie, accès limité aux soins).

\section{Coqueluche [2]}

Vaccination recommandée de base (adultes) Une dose unique de vaccin contre la coqueluche $\left(\mathrm{p}_{\mathrm{a}}\right)$ (rappel ou primovaccination) est recommandée à tous les adultes âgés de 25 à 29 ans (jusqu'au 30e anniversaire) en utilisant un vaccin combiné dTpa. L'intervalle minimum depuis la dernière vaccination contre le tétanos doit être en principe de 2 ans.

Vaccinations recommandées pour des situations à risque (adolescents/adultes)

Une dose unique de vaccin contre la coqueluche est recommandée à toutes les personnes (adolescents, adultes), quel que soit leur âge, en contact régulier familial ou professionnel, actuel ou dans un futur proche avec des nourrissons de moins de 6 mois (risque accru de complications). Ces personnes devraient être vaccinées dès que possible contre la co-

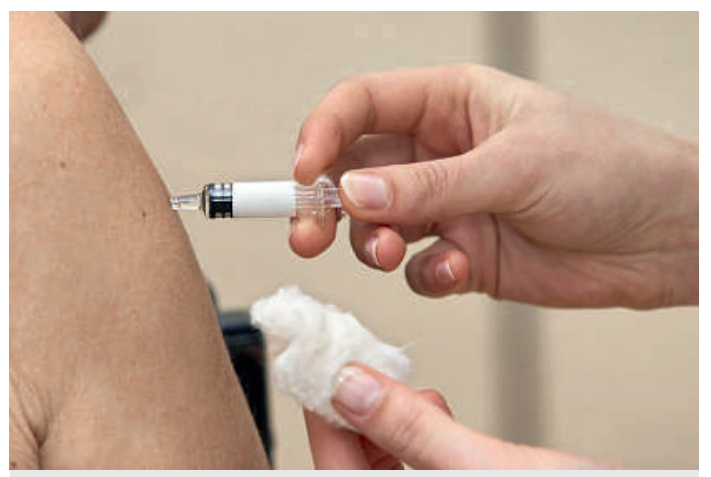

Les intervalles entre les rappels de vaccination ont changé.

queluche si elles n'ont pas été vaccinées contre cette maladie durant les 10 années précédentes (maximum 1 dose à l'âge adulte). Dans cette situation, l'intervalle minimum depuis la dernière dose de vaccin antitétanique est seulement de 4 semaines.

Le but est d'offrir une protection aux adultes contre la coqueluche et par conséquent de diminuer le risque d'infection chez les nourrissons.

HPV [3]

Vaccination recommandée de base

De nouvelles données scientifiques indiquent que lorsque la première dose de vaccin contre les HPV est administrée avant le $15^{\mathrm{e}}$ anniversaire, deux doses suffisent. Dorénavant un schéma vaccinal de 2 doses est recommandé pour les adolescentes âgées de 11 à 14 ans. L'intervalle entre les doses est de 6 mois (minimum 4 mois). Lorsque la vaccination débute à 15 ans ou plus, le schéma de vaccination de 3 doses (0,1-2, 6 mois) reste indiqué. Cette nouvelle recommandation concerne les deux vaccins contre les HPV autorisés en Suisse.

\section{Méningocoques [4]}

Vaccinations recommandées pour des groupes/ situations à risque

Le vaccin conjugué quadrivalent contre les méningocoques A, C, W135, Y (MCV-ACWY; Menveo $\left.{ }^{\circledR}\right)$, récemment mis sur le marché, est recommandé pour la (primo-)vaccination des personnes à risque accru de maladies invasives à méningocoques ou des personnes à risque accru d'exposition (personnel de laboratoire, prophylaxie post-expositionnelle aux types 
A, W135 ou Y, voyageurs) à partir de l'âge de 12 mois (utilisation hors autorisation entre 12 mois et 11 ans). Schéma de vaccination:

- 2 doses à 4-8 semaines d'intervalle pour les personnes à risque accru d'infection invasive, 1 dose pour les autres situations à risque, suivies de rappels par le vaccin quadrivalent polysaccharidique (MPV-ACWY) à intervalle de 5 ans si le risque persiste.

- Les personnes ayant été vaccinées par le vaccin MPV-ACWY plus de 5 ans auparavant devraient recevoir 1 dose de MCV-ACWY. Les rappels suivants seront à nouveau effectués avec MPVACWY, tous les 5 ans si le risque persiste.

\section{Rage [5]}

\section{Vaccinations recommandées pour des situa- tions à risque}

Il est recommandé de réduire le nombre de doses de 5 à 4 dans le cas d'une prophylaxie post-expositionnelle appliquée dans les règles (traitement des plaies, administration d'immunoglobulines antirabiques (Ig), et vaccination aux jours $0,3,7,14$ et présence à 21 jours d'un taux d'anticorps protecteur).

\section{Information supplémentaire}

En annexe de ce numéro: la fiche d'information «Plan de vaccination suisse» (état février 2012). Il s'agit d'un résumé du «Plan de vaccination suisse 2012» de la série Directives et Recommandations. Cette fiche existe en trois langues: français, allemand et italien. Des exemplaires supplémentaires peuvent être commandés gratuitement à l'adresse suivante: OFCL, Dis- tribution des publications fédérales, Case postale, 3003 Berne, fax 03132550 58, e-mail: verkauf. zivil[at]bbl.admin.ch; Internet: www.bundespublikationen.admin.ch, no. de commande: 311.267.f (d, i).

Le plan de vaccination suisse 2012 (version complète) peut être téléchargé sur les sites Internet de l'OFSP (www.sevacciner.ch) ou d'InfoVac (www.infovac.ch). En outre, chacun peut vérifier son statut vaccinal grâce au carnet de vaccination électronique et s'inscrire pour recevoir une notification lorsqu'un rappel est nécessaire, offres disponibles sur le site Internet www.mesvaccins.ch.

\section{Références}

1 Office fédéral de la santé publique, Commission fédérale pour les vaccinations. Plan de vaccination suisse. Directives et recommandations. Berne; 2012.

2 Office fédéral de la santé publique, Commission fédérale pour les vaccinations. Optimisation des rappels vaccinaux contre la diphtérie, le tétanos et la coqueluche (dT/dTpa) chez l'adulte. Bull OFSP. 2011; $\mathrm{N}^{\circ}$ 51: 1161-71.

3 Office fédéral de la santé publique, Commission fédérale pour les vaccinations. Vaccination contre les HPV: passage du schéma à trois doses au schéma à deux doses chez les adolescentes âgées de moins de 15 ans. Bull OFSP. 2012; N 6:106-10.

4 Office fédéral de la santé publique, Commission fédérale pour les vaccinations. Mise à jour des recommandations de vaccination contre les méningocoques: introduction d'un vaccin quadrivalent conjugué. Bull OFSP. 2011; N 34:711-7.

5 Office fédéral de la santé publique. Changement du schéma de prophylaxie postexpositionnelle contre la rage: mise à jour des recommandations. Bull OFSP. 2012; N6:111-5.

\section{Tableau}

Vaccinations recommandées de base et complémentaires (CFV/OSFP).

\begin{tabular}{|c|c|c|c|c|c|c|c|c|c|c|c|c|}
\hline \multirow[b]{2}{*}{ Age ${ }^{1)^{*}}$} & \multicolumn{9}{|c|}{ Vaccinations recommandées de base } & \multicolumn{3}{|c|}{ Vaccinations complémentaires } \\
\hline & DTP 2) & Polio 2) & Hib & HBV ${ }^{8)}$ & ROR & HPV & VZV & Grippe & $\begin{array}{l}\text { Pneumo- } \\
\text { coques }\end{array}$ & $\begin{array}{l}\text { Pneumo- } \\
\text { coques }\end{array}$ & Méningocoques C & HPV \\
\hline Naissance & & & & 9) & & & & & & & & \\
\hline 2 mois & $\mathrm{DTP}_{\mathrm{a}}$ & IPV & $\mathrm{Hib}^{6)}$ & 10) & & & & & & PCV13 20) 21) & & \\
\hline 4 mois & $\mathrm{DTP}_{\mathrm{a}}$ & IPV & $\mathrm{Hib}$ & 10) & & & & & & PCV13 & & \\
\hline 6 mois & $\mathrm{DTP}_{\mathrm{a}}$ & IPV & $\mathrm{Hib}$ & 10) & & & & & & & & \\
\hline 12 mois & & & & & ROR & & & & & PCV13 21) & & \\
\hline $12-15$ mois & & & & & & & & & & & MCV-C 22) & \\
\hline $15-24$ mois & $\mathrm{DTP}_{\mathrm{a}}$ & IPV & $\mathrm{Hib}^{7)}$ & 10) & ROR & & & & & & & \\
\hline $4-7$ ans & $\mathrm{DTP}_{\mathrm{a}}$ & IPV & & & 13) & & & & & & & \\
\hline $11-14 / 15$ ans & $\mathrm{dT} /\left(\mathrm{dT} \mathrm{p}_{\mathrm{a}}\right)^{3)}$ & 5) & & $\mathrm{HBV}^{11)}$ & 13) & HPV 15) 16) & VZV ${ }^{17)}$ & & & & MCV-C 23) & \\
\hline $25-29$ ans & $\mathrm{dTp} \mathrm{p}^{4)}$ & 5) & & 12) & 14) & & 17) & & & & & HPV 24) \\
\hline 45 ans & dT ${ }^{4)}$ & 5) & & 12) & 14) & & & & & & & \\
\hline$\geq 65$ ans & dT 4) & 5) & & 12) & & & & 18) & 19) & & & \\
\hline
\end{tabular}

* 1)-24) cf. document original «Plan de vaccination suisse» sur www.sevacciner.ch ou www.infovac.ch. 